\title{
Flow-Through Cell Apparatus (USP Apparatus 4): Operation and Features
}

Nikoletta Fotaki

e-mail:n.fotaki@bath.ac.uk

Department of Pharmacy \& Pharmacology, University of Bath, Claverton Down, BA2 7AY, Bath, UK

\section{INTRODUCTION}

D issolution testing plays an important role in several areas during drug development. It can be used as a quality control tool to monitor batch-to-batch consistency of drug release from a dosage form and as an in vitro surrogate for in vivo performance that can guide formulation development and ascertain the need for bioequivalence tests. Several apparatus (compendial and noncompendial) are used for the study of dissolution of compounds and dosage forms.

The flow-through cell method for the study of dissolution first appeared in 1957 as a flowing medium dissolution apparatus developed by FDA (1). The method was adapted by USP, the European Pharmacopoeia (Ph.Eur.), and the Japanese Pharmacopoiea (JP), and the flowthrough cell became an official apparatus (Apparatus 4 for the USP and Ph. Eur., Apparatus 3 for JP). Specifications and methodology are described in the relevant chapters of the pharmacopeias-USP Chapter $<711>$ Dissolution (2), Ph. Eur. 2.9.3 (3), and JP XV, 6.10 Dissolution Test (4)—and there is good harmonization among them.

\section{DESCRIPTION AND OPERATION OF THE SYSTEM}

The system consists of a reservoir containing the dissolution/release medium, a pump that forces the medium upwards through the vertically positioned flow-cell, and a water bath to control the temperature in the cell.

\section{Dissolution and Release Media}

Conventional buffers, media proposed by the pharmacopeias, and biorelevant media can be used. Media can be changed during the experiment (a medium selector can be used). Dissolution media may need to be deaerated.

\section{Flow-Through Cell}

Different types of cells are available for testing tablets, powders, suppositories, hard- and soft-gelatin capsules, implants, semisolids, suppositories, and drug-eluting stents. For orally administered solid dosage forms, two different cells are described (Figure 1): the large cell (22.6-mm i.d.) and the small cell (12-mm i.d.) that provide approximate volumes of $19 \mathrm{~mL}$ and $8 \mathrm{~mL}$, respectively, for dissolution (cell volumes without glass beads). Usually the bottom cone of the cell is filled with small glass beads (about 1 -mm diameter), and one bead (about 5-mm diameter) is positioned at the apex to prevent material from descending into the inlet tubing. Different amounts of small glass beads can be used according to the experimental setup. The sample can be placed upon a holder but also can be placed on or within the glass-bead bed. For dispersed systems (i.e., suspensions, powders), mixing of the sample within the glass-bead bed has also been reported.

\section{Pump and Flow Patterns}

Peristaltic and pulsating piston pumps can be used; the latter is more common. Usually a sinusoidal pulse rate of $120 \pm 10$ pulses per minute is used. The pulse rate remains constant independent of the selected flow rate, and the need for further stirring is eliminated due to the pulsating pattern of the pump. USP, Ph. Eur., and JP have harmonized on the possibility that the pump is pulseless (USP 33). Linear flow velocity defines the hydrodynamics in the cell; different cell diameters are associated with axial velocities, which correspond to the flow rate (5). Flow rates over a wide range can be used (according to the specifications of the pump). Typical flow rates are 4,8 , and $16 \mathrm{~mL} / \mathrm{min}$, and usually flow should be maintained at $\pm 5 \%$ of the nominal value (regular calibration of the pump is recommended).

The pattern is described as "turbulent" when operated without glass beads in the entry cone (usually required for samples that need a higher agitation rate to release its active, i.e., implants), and "laminar" when glass beads are used. A laminar flow is characterized by fluid particles moving in parallel to one other in the flow direction, and a turbulent flow is characterized by the rapid movement of fluid particles in all direction within the flow direction (6). Recently, characterization of the flow pattern inside the cell with the use of magnetic resonance imaging (MRI) revealed that the flow field inside the cell is mainly heterogeneous rather than fully developed laminar flow and is characterized by recirculation and backward flow (7). The use of 1-mm beads distributes the flow but does not ensure a fully developed laminar flow profile. It has been proposed to label the operational modes in the flowthrough cell in terms of their physical configurations. The designation "open column" and "packed column" instead of "turbulent mode" and "laminar mode" of operation have been suggested (6).

\section{Open and Closed Mode}

The flow-through cell apparatus can operate in two different modes: (1) as an open system with fresh solvent from the reservoir continuously passing through the cell 


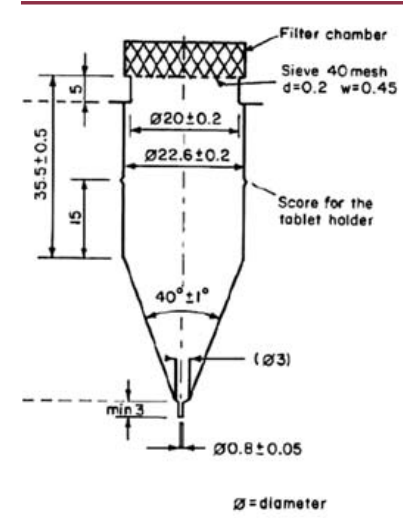

A)

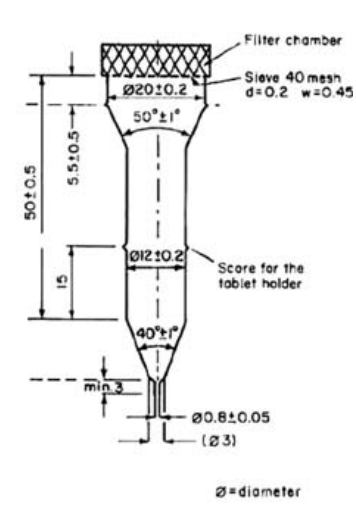

B)
Figure 1. (A) Large and (B) small flow-through cell for orally administered solid dosage forms. Reprinted from ref 2. Copyright 2011 The United States Pharmacopeial Convention.

(Figure 2) and (2) as a closed system (Figure 3) where a fixed volume of liquid is recycled. The open system is selected for samples that require high volume of media (i.e., low solubility compounds), and the closed system is selected when a low volume of medium is required.

\section{Filtration}

A filter is positioned at the inner top of the cell to retain undissolved material (5). Usually glass fiber filters are used (single or combination of different pore sizes). The use of glass wool is sometimes suggested for dosage forms with insoluble and/or sticky particles. Appropriate selection of the filter is required for efficient filtration and to avoid backpressure created by filter resistance.

\section{Sampling}

The collected samples can be analyzed directly by a UVvis spectrophotometer or a fiber-optic probe, or they can be collected in fractions and analyzed by HPLC or other appropriate method. Sample collection can be automated to ensure consistency. On-line automation, in which the sample is measured by UV, or off-line automation, in which the sample is collected automatically and analyzed afterwards with an appropriate method, can be used.

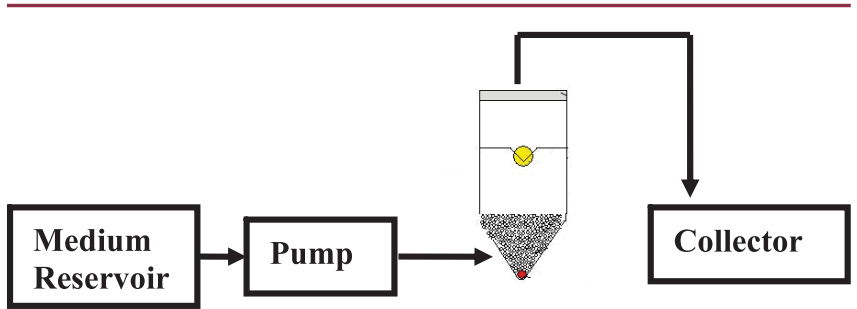

Figure 2. Schematic diagram of the open-loop configuration for the flow-through cell apparatus.

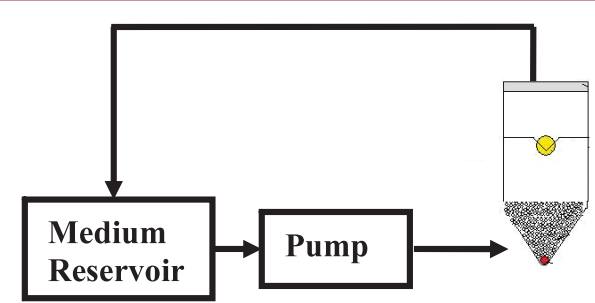

Figure 3. Schematic diagram of the closed-loop configuration for the flow-through cell apparatus.

\section{Data Collection}

When the system operates in the open mode, the data collected represents the amount dissolved/released at specific time intervals (estimate of release rate) and is in noncumulative form (8). Data can be transformed to the cumulative form; in this case, any mistakes associated with the estimation of the total drug released during a specific time interval will be transferred to the next time interval. If a model is to be fitted to the data, by converting them to the cumulative form, the fundamental assumption of independence of errors is violated (5). An example of data collected during a dissolution/release experiment in noncumulative form and then transformed to cumulative form when the system operates in the open mode is presented in Figure 4. Data collected when the system operates in the closed mode is in cumulative form.

\section{FEATURES OF THE FLOW-THROUGH CELL SYSTEM}

The flow-through cell system has several characteristics that can offer important information for the study of compounds and dosage forms (5):

- Medium and/or flow rate can be changed within a single run. Study of formulation release patterns (i.e., release from controlled-release formulations), targeted delivery, and study of release under fasted- or fed-state conditions are facilitated. Carry-over effects during the experiment can be determined, and testing of formulation robustness is feasible.

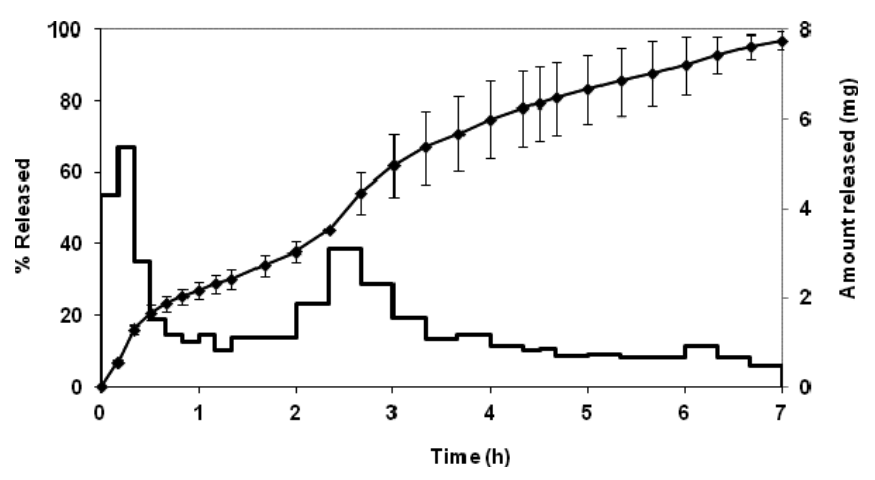

Figure 4. Dissolution/release data collected using the flow-through cell apparatus in noncumulative form and transformed to cumulative form. 
- Sink conditions can be maintained, due to the continuous flow of fresh medium, when the system operates in the open-loop configuration. This feature is important for the study of poorly soluble drugs, in cases where traditional closed systems do not allow the provision of sink conditions for these drugs. The dissolution rate reflects the behavior of the sample and not the solubility of the substance, as in the closed systems.

- Study of samples with low drug loading is feasible when the system operates in the closed-loop configuration, as small volume of medium can be used.

- The system can be used for the characterization of apparent dissolution, as samples (powder) can be placed in the cell without application of mechanical forces (i.e., compression).

- Release from dosage forms over extended periods can be studied, as this setup eliminates the evaporation issue that can be observed with other apparatus.

- The flow-through cell offers a controlled hydrodynamic environment, and intralumenal hydrodynamics are more efficiently simulated compared with other in vitro setups.

- The hydrodynamics inside the cell are not affected by media change and sampling, as can occur in traditional closed systems (i.e., rotating paddle apparatus, rotating basket apparatus).

- Development of in vitro-in vivo correlations can be easier as a single profile corresponding to the release of the drug in the entire gastrointestinal tract can be obtained when the media change is applied.

- Different sample types and dosage forms can be studied (as several cell types are available).

\section{POINTS TO CONSIDER \\ Filtering Issues}

Filtering issues (blocking, clogging) that affect the maintenance of desired flow rates and create back up pressure problems can occur in some cases (i.e., experiments with powders, use of heterogeneous media, tests for significant periods of time) $(5,6)$.

\section{Performance Verification Test (PVT)}

No specific USP calibrator tablets for the flow-through cell apparatus exist. Several studies have been performed using the USP calibrator tablets to prove the correct handling of the instrument and the correct treatment of data $(9,10)$. Currently, there is no PVT for dissolution procedures that relies on the flow-through cell apparatus. A PVT is a means of assessing the integrity of the overall procedure, including not only the apparatus, but also the analytical procedure and the analyst. Recently, Eaton et al. (11) have shown that salicylic acid tablets are a suitable reference material for the PVT for this apparatus.

\section{Combination with Other Systems}

Studies have shown the feasibility of combining the flow-through cell system with other systems, for enhancing knowledge of dissolution and performance of dosage forms. For example, integration of the flow-through cell apparatus with MRI system has been proposed for discriminative evaluation of controlled-release dosage forms with similar dissolution profiles by investigating physical changes in the dosage form that can aid tablet formulation and can be used as a potential Process Analytical Technology tool $(12,13)$. Furthermore, the flowthrough cell system can be used to detect the influences of dosage forms on permeation processes by connection to cell-culture-based permeability assessment (14).

\section{REFERENCES}

1. Langenbucher, F.; Benz, D.; Kurth, W.; Moller, H.; Otz, M. Standardized Flow-Cell Method as an Alternative to Existing Pharmacopoeial Dissolution Testing. Pharm. Ind. 1989, 51, 1276-1281.

2. The United States Pharmacopeia and National Formulary USP 32-NF 27; The United States Pharmacopeial Convention, Inc.: Rockville, MD, 2009.

3. European Pharmacopoeia, 7th ed.; European Directorate for the Quality of Medicines \& Healthcare, Council of Europe: Strasbourg, France, 2011.

4. Japanese Pharmacopoeia, 15th ed.; Ministry of Health, Labour, and Welfare:Tokyo, Japan, 2006.

5. Fotaki, N.; Reppas, C. The Flow Through Cell Methodology in the Evaluation of Intralumenal Drug Release Characteristics. Dissolution Technol. 2005, 12 (2), 17-21.

6. Kakhi, M. Classification of the flow regimes in the flow-through cell. Eur. J. Pharm. Sci. 2009, 37, 531-544.

7. Shiko, G.; Gladden, L. F.; Sederman, A.J.; Connolly, P.C.; Butler, J.M. MRI studies of the hydrodynamics in a USP 4 dissolution testing cell.J.Pharm. Sci. 2011, 100, 976-991.

8. Brown, W. Apparatus 4 Flow-Through Cell: Some Thoughts on Operational Characteristics. Dissolution Technol. 2005, 12 (2), 28-30.

9. Nicklasson, M.; Langenbucher, F. Description and Evaluation of the Flow Cell Dissolution Apparatus as an Alternative Test Method for Drug Release. Pharm. Forum 1990, 16, 532-540.

10. Krämer, J.; Stippler, E. Experiences with USP Apparatus 4 Calibration. Dissolution Technol. 2005, 12 (2), 33-39.

11. Eaton, J.W.;Tran, D.; Hauck, W.W.; Stippler, E.S. Development of a Performance Verification Test for USP Apparatus 4. Pharm. Res. [Online early access]. DOI: 10.1007/s11095-011-0559-6. Published Online: Aug 9, 2011.http://www.springerlink.com/content/ h721245206531508/fulltext.html (accessed Oct 18, 2011).

12. Dorożyński, P.; Kulinowski, P.; Mendyk, A.; Młynarczyk, A.; Jachowicz, R. Novel Application of MRI Technique Combined with Flow-Through Cell Dissolution 
Apparatus as Supportive Discriminatory Test for Evaluation of Controlled Release Formulations. AAPS PharmSciTech 2010, 11, 588-597.

13. Nott, K.P. Magnetic resonance imaging of tablet dissolution. Eur. J. Pharm. Biopharm. 2010, 74, 78-83.
14. Motz, S. A.; Schaefer, U. F.; Balbach, S.; Eichinger, T.; Lehr, C.-M. Permeability assessment for solid oral drug formulations based on Caco-2 monolayer in combination with a flow-through dissolution cell. Eur. J. Pharm. Biopharm. 2007, 66, 286-295. 